\title{
Oral cancer screening practices of oral health professionals in Australia
}

Rodrigo Mariño ${ }^{1 *}$, Satoru Haresaku², Roisin McGrath¹, Denise Bailey ${ }^{1}$, Michael Mccullough ${ }^{3}$, Ross Musolino ${ }^{4}$, Boaz Kim ${ }^{5}$ Alagesan Chinnassamy ${ }^{5}$ and Michael Morgan ${ }^{1}$

\begin{abstract}
Background: To evaluate oral cancer-related screening practices of Oral Health Professionals (OHPs - dentists, dental hygienists, dental therapists, and oral health therapists) practising in Victoria, Australia.

Methods: A 36-item survey was distributed to 3343 OHPs. Items included socio-demographic and work-related characteristics; self-assessed knowledge of oral cancer; perceived level of confidence in discussing oral health behaviors with patients; oral cancer screening practices; and self-evaluated need for additional training on screening procedures for oral cancer.
\end{abstract}

Results: A total of 380 OHPs responded this survey, achieving an overall response rate of $9.4 \%$. Forty-five were excluded from further analysis. Of these 335 OHP, 72\% were dentists; $(n=241)$; either GDP or Dental Specialists; 13. $7 \%(n=46)$ were dental hygienists; $12.2 \%(n=41)$ were oral health therapists, and the remaining $2.1 \%(n=7)$ were dental therapists. While the majority (95.2\%) agreed that oral cancer screening should be routinely performed, in actual practice around half (51.4\%) screened all their patients. Another 12.8\% "Very rarely" conducted screening examinations. The probability of routinely conducting an oral cancer screening was explored utilising Logistic Regression Analysis. Four variables remained statistically significant $(p<0.0001)$. Results indicate that the likelihood of conducting an oral cancer screening rose with increasing levels of OHPs' confidence in oral cancer-related knowledge $(\mathrm{OR}=1.35 ; 95 \% \mathrm{Cl}: 1.09-1.67)$ and with higher levels of confidence in discussing oral hygiene practices with patients $(\mathrm{OR}=1.25 ; 95 \% \mathrm{Cl}: 1.03-1.52)$. Results also showed that dental specialists were less likely to perform oral cancer screening examinations compared with other OHPs $(\mathrm{OR}=0.18 ; 95 \% \mathrm{Cl}$ : 0.07-0.52) and the likelihood of performing an oral cancer screening decreased when the "patient complained of a problem" (OR=0.21; 95\% Cl: $0.10-0.44)$.

Conclusion: Only half the study sample performed oral cancer screening examinations for all of their patients. This study provides evidence of the need for further oral cancer-related education and screening training for OHPs, which is vital to enhance oral cancer prevention and early detection.

Keywords: Oral cancer, Screening practices, Self-confidence, Oral health professionals, Australia

\section{Background}

Globally, cancers are the second most common cause of death with about one in every six deaths attributable to cancer [1]. In 2012, there were 14.1 million new cancer cases and 8.2 million cancer deaths worldwide [1]. Over the last few years, despite advances in early diagnosis and treatment, and in the survival rate, there has been an increase in the overall incidence of cancers [2]. The

\footnotetext{
* Correspondence: r.marino@unimelb.edu.au

${ }^{1}$ Oral Health Cooperative Research Centre, Melbourne Dental School,

University of Melbourne, Melbourne, VIC 3010, Australia

Full list of author information is available at the end of the article
}

cost of cancer treatment has also increased with advancements in early diagnosis and treatment [2]. Among Head and Neck Cancers (HNC), the most commonly occurring are Oro-Pharyngeal Cancers (OPC), which include the lip, oral cavity, and oropharynx. Globally, OPC are the sixth most common malignancy [3]. Despite the technological advancement, cancer awareness and improvement in survival rates for some cancers [2], no significant improvement has been reported in the five-year survival rates for OPC $[4,5]$.

OPC is one of the few oral diseases encountered by the oral health team that has significant morbidity and 
premature mortality Tobacco products, alcohol consumption and sun exposure are the most recognized risk factors for OPC [6]. But the incidence of Human Papilloma Virus (HPV) related OPC has also been on the rise, particularly in younger adults who have never smoked or used other tobacco products [7, 8]. In Australia, HNC are the seventh most common cancers with an incidence rate of 17 cases per 100,000 persons [9]. In 2017, estimated deaths due to HNC is well over 1000 individuals (with three fourths of the reported deaths in males), that amounts to roughly $3.7 \%(n=4956)$ of all new cancers diagnosed [9].

A recent effectiveness review on oral cancer screening, demonstrated conventional oral examination to be a feasible and satisfactory option for opportunistic screening in dental settings with sensitivity and specificity similar to breast and cervical cancer screening programs [4, 10-12]. Early diagnosis of OPC can greatly increase the five-year survival rates from $50 \%$ to more than $80 \%[6,13]$.

$\mathrm{OPC}$ are known to be amenable to early detection as they primarily occur at sites that are accessible and visible during a non-invasive examination [4]. They are often preceded by a visible precancerous lesion enabling early detection and treatment [14]. The tumors' proliferative factor and stage at the time of diagnosis largely determines the prognosis of cancers [15]. However, only $30 \%$ of OPC are identified at an early stage [16] with the majority (50\%) being diagnosed at an advanced stage of metastasis (stage III or IV). This is largely due to late presentation, delayed diagnosis and lack of a clear referral pathway between doctors and dentists [17-21]. This forms the strongest argument for early diagnosis of OPC and initiating early treatment.

It is essential that OHPs such as dentists, dental hygienists (DHs), dental therapists (DTs), and oral health therapists (OHTs), understand the importance of conducting a thorough oral screening examination for malignant and potentially-malignant lesions as part of their routine clinical assessments, even in younger populations originally considered at lower risk for oral cancer $[22,23]$. The World Dental Federation and Dental Associations, including the Australian Dental Association, proactively encourage Oral Health Professionals (OHP) to incorporate oral mucosal examinations as part of routine assessment [6, 24-27]. Several studies have assessed dentists' knowledge, attitudes and practices regarding oral cancer [28-33]. However, only a few studies include DH, DT, and OHT. Thus, clinical screening practices regarding oral cancer among the complete range of oral health professionals remain largely unknown.

Screening programs should be designed to detect precancerous lesions or malignancies in their asymptomatic phases for all patients, not only in the older population groups, traditionally known to be at higher risk, but also in younger patient groups amongst which there has been a rising incidence of $\mathrm{OPC}[4,7,8,34]$. Nonetheless, studies among oral health professionals in Australia identified lack of training, lack of confidence and time constraints as the three most important barriers for oral cancer screening $[4,21]$. Expansions of early diagnosis and treatment of oral cancers should be a priority from a public health viewpoint.

The purpose of this study was to investigate oral cancer screening practices of OHPs in Victoria, Australia as well as evaluate their oral cancer-related opinions and attitudes; and to identify factors associated with the likelihood of an OHP performing an oral cancer screening examination. This information can be used to identify gaps in oral cancer knowledge among OHPs, and subsequently to inform the development of continuing education programs specifically focused on oral cancer prevention, identification and management of malignant and potentially-malignant lesions of the oral cavity and oropharynx at the state level.

\section{Methods}

The study was a cross-sectional survey of OHP in Victoria, Australia. As of September 2014, there were 4781 registered OHPs in Victoria, which amounts to $23 \%$ of the total Australian dental work force. Of them, 3715 are dentists and another 585 are registered DH/DT/OHTs in Victoria [35]. With the approval of the Human Research Ethics Committee at the University of Melbourne, a request was submitted to the professional associations representing Victorian OHPs, including the Australian Dental Association, the Dental Hygienists Association of Australia (Victorian Branch) and the Victorian Dental and Oral Health Therapists Association, to distribute the survey to their members. OHPs were initially contacted by mail in September 2014. Two weeks and four weeks after the first mailing, postcards were sent to thank those who had returned the questionnaire and to remind the others of the importance of the study, and encourage them to complete the survey. In an attempt to maximize the response rate, participants were offered a choice between completing either a paper-and-pencil survey or a web-based survey (i.e. invitation-letter with URL of online survey, questionnaire and reply paid envelope). Additionally, after the initial postal contact, the Australian Dental Association indicated a preference that e-mails be sent with the link to the online survey, as a reminder. Two reminders were sent in March and April 2015.

According to the literature [36-38] the expected response rate among OHPs is around $30 \%$. However, an Australian study involving medical doctors, reported a mean response rate of $19.7 \%$ when using a similar simultaneous mixed-mode survey (a paper questionnaire and login details sent together) [39]. Therefore, we expected a final sample size of around 611 dentists and 89 dental 
hygienists/dental therapists/oral health therapists. The data collection period was from September 2014 to March 2015.

This sample size would be large enough to conduct the required statistical analyses as the minimum sample size necessary to study the relationship between a dependent variable (e.g., knowledge of risk factors for oral cancers) and a set of independent variables based on the case of 10 independent variables accounting for $5 \%$ of the variance (a conservative estimate) in the dependent variable, indicates that a sample size of 335 will yield a power of 0.80 , that is, an $80 \%$ chance of explaining that proportion of the variance at a $p$-value of 0.05 [40].

Survey data was derived from a questionnaire consisting of four parts: socio-demographic and work characteristics (6 items); knowledge of risk factors for oral cancers (14 items); level of confidence in discussing health behaviors with patients (5 items); and oral cancer screening practices (11 items). The socio-demographic information included sex, age-range and location of practice. By occupational sub-stratification, participants were classified into three professional groups: 'Dentists'; 'Dental Hygienists (DH)'; and 'Oral Health Therapists and Dental Therapists (OHT/ DT)'. Dentists were further sub-divided into General Dental Practitioners (GDP) and 'Dental Specialists'. Professional experience was classified into six categories: '5 years or less'; ' 6 to 10 years'; ' 11 to 15 years'; ' 16 to 20 years'; ' 21 to 25 years'; and 'More than 25 years'. Employment history information included the postal code(s) of their main geographical location of practice. Using the Australia Post's local delivery service guidelines, work locations were classified as being 'Urban', or 'Rural' [41].

Participants were asked to self-assess their level of knowledge about risk for oral cancer, utilizing a 10-point numerical scale $(0=$ 'Very poor' to $10=$ 'Excellent'). Knowledge regarding risk factors for oral cancer included 11 factors (smoking, periodontal disease, caffeine consumption, family history of oral cancer, chewing betel nut, human papillomavirus [HPV] infections, hepatitis $\mathrm{C}$ infection, chewing tobacco, level of alcohol consumption, herpes simplex virus infections and history of oral cancer). Participants were asked to select which of the 11 factors were risk factors for oral cancers utilising a three-option response of 'Yes, 'No' or 'Unsure'. To quantify the degree of knowledge of oral cancer risk factors among OHPs, a score system was developed, which involved adding the weighted answers for each of the 11 questions on risk factors. Scores ranged from 0 to 9 . OHPs were also asked two questions regarding their perceived need for more training and education on oral cancers with response categories of 'Yes'; 'No'; or 'Unsure'.

Questions related to oral cancer screening attitudes and practices asked: a) whether OHP should routinely screen for oral cancer; b) self-reported frequency of screenings (response options were: 'Very rarely'; 'With less than 50\% of patients'; 'With $50 \%$ or more of patients'; and 'With every patient'); c) factors that prompt OHPs to initiate discussion of risk factors with patients; d) age groups routinely screened for oral cancer; e) whether OHPs inform the patient about being screened; f) if patients are informed what the screening involves; g) if they have ever used additional diagnostic tools to detect malignant lesions; and h) their course of action when an abnormal lesion was detected. Participants were also asked whether they had ever referred a patient to an oral medicine specialist.

Items focusing on OHPs' level of confidence in discussing health behaviors with patients, utilized a numerical 10 -point scale $(0=$ not at all confident to $10=$ completely confident). These behaviors included; tobacco use, alcohol consumption, sexual behaviors, oral hygiene practices and diet and nutrition.

\section{Data analysis}

The analysis provides descriptive information on the participants' work and various socio-demographics. Bivariate associations were evaluated with Chi-squared analysis for nominal or ordinal variables. For variables on an interval scale, results were analyzed using one-way analysis of variance (ANOVA). A significant ANOVA was followed by post-hoc comparisons using Tukey's Honestly Significant Differences tests. To better understand the association between the combination of socio-demographic, work and psychosocial variables and the probability of conducting a screening exam, a stepwise logistic regression analysis (LRA) was performed. All $p$-values $<0.05$ were considered significant. Data manipulation and analysis were conducted using IBM SPSS Statistics (Version 21.0, IBM Corporation, Endicott, NY, USA).

\section{Results}

A total of 380 OHPs responded, achieving an overall response rate of $9.4 \%$ (ranging from $9.3 \%$ among dentists to $22.9 \%$ among DH/DT/OHTs). Fourteen respondents indicated that they do not practice dentistry anymore while another 31 mentioned that they do not routinely treat adult patients. These 45 OHPs were excluded from further analysis. Thus, a total of 335 were included in the final analysis. Most this group were dentists $(72 \% ; n=241)$, either GDP $(63.6 \%)$ or Dental Specialists (8.4\%). Another $13.7 \%(n=46)$ were DHs; $12.2 \%(n=41)$ were OHTs, and the remaining $2.1 \%(n=7)$ were DTs. By gender, the majority were female (58.2\%). Among dentist participants, a marginally higher percentage were male $(55.8 \%)$, while more than $90 \%$ of DH/DT/OHTs were female.

By age, more than half the total participants (55.5\%) were 45 years or younger; however, the largest age group was the ' 46 to 55 years old' group (24.8\%), with $10.7 \%$ of the participants in the ' 25 years of age or younger' group. 
Differences in age and gender were statistically significant by oral health profession $(p<0.001)$. Those working exclusively as OHT were younger than those working as dentists or DHs (See Table 1).

When participants were asked about the location of their workplace, the majority (76.4\%) indicated an 'Urban' location, with no statistically significant difference by professional background. Regarding the length of time practicing as an OHP, $33.4 \%$ indicated more than 25 years of practice; $30.8 \%$ between 11 and 25 years; $23.0 \%$ reported five years or less of practice; and the remaining 12.8\% reported between 6 and 10 years of practice. As expected, differences by duration of practice between groups were statistically significant $(P<0.001)$.

Oral cancer screening examination.

Almost all OHPs (95.2\%) indicated the importance of and need for routine oral cancer screening in their clinical practice. However, only half of those surveyed (51.3\%) reported conducting a comprehensive oral cancer screening for all of their patients (Table 2). Another 21.8\% reported conducting a comprehensive oral cancer screening for ' $50 \%$ or more' of their patients. OHPs who reported performing oral cancer screening in 'less than 50\% of patients' or 'Very Rarely' comprised $14.0 \%$ and $12.8 \%$ of respondents, respectively. Differences by professional background for

Table 1 Demographic, work characteristics of oral health professionals in Victoria

\begin{tabular}{lllll}
\hline & $\begin{array}{l}\text { Dentists } \\
(n=242)\end{array}$ & $\begin{array}{l}\text { DHs } \\
(n=45)\end{array}$ & $\begin{array}{l}\text { OHT/DTs } \\
(n=48)\end{array}$ & $\begin{array}{l}\text { Total } \\
(n=335)\end{array}$ \\
\hline Age of group & & & & $*$ \\
25 or less & 7.4 & 2.1 & 35.5 & 10.7 \\
$26-35$ & 19.0 & 11.1 & 50.0 & 22.4 \\
36-45 & 23.2 & 35.6 & 6.2 & 22.4 \\
46-55 & 25.2 & 42.3 & 6.2 & 24.8 \\
$>55$ & 25.2 & 8.9 & 2.1 & 19.7 \\
Gender & & & & $*$ \\
Male & 55.8 & 2.2 & 8.3 & 41.8 \\
Female & 44.2 & 97.8 & 91.7 & 58.2 \\
Duration of practicing & & & $*$ \\
5 or less & 15.7 & 11.1 & 80.5 & 23.0 \\
6-10 & 12.4 & 11.1 & 17.1 & 12.8 \\
11-15 & 11.6 & 13.3 & 2.4 & 11.0 \\
16-20 & 9.9 & 15.6 & 0 & 9.9 \\
21-25 & 9.9 & 20.0 & 0.0 & 9.9 \\
$>$ 25 & 40.5 & 28.9 & 0.0 & 33.4 \\
Location of workplace & & & \\
Urban & 76.6 & 77.3 & 74.5 & 23.6 \\
Rural & 23.4 & 22.7 & 25.5 & \\
\hline *Chi- squared test: $p$-value: 0.001 & & & \\
\hline
\end{tabular}

*Chi- squared test; $p$-value: 0.001 completing oral health screening were not statistically significant. The vast majority of respondents $(92.2 \%)$ routinely screened patients 40 years of age or older for oral cancer. This proportion was $68.4 \%$ for $20-39$ years old patients, and $38.5 \%$ for patients younger than 20 years of age.

Among those OHPs who reported not conducting a cancer screening for all their patients $(n=193)$, the decision to screen was influenced by the patient complaining of an oral health problem (65.6\%); the patient's age (63.8\%); or the patient's medical history (53.3\%). Another $35.0 \%$ of respondents indicated other factors influencing their decision to conduct an oral cancer screening examination, such as exposure to risk factors (i.e. alcohol consumption and smoking). Of note, only the 'age of the patient' as an influencing variable reached statistical significance $(p<0.05)$.

When excluding those who 'Very rarely' conducted an oral cancer screening examination on their patients, just over half of respondents (51.9\%) indicated that they 'Sometimes' informed the patient that they were screening for oral cancer lesions. Another 19.9\% 'Always' informed patients that they were screening for potentially malignant or malignant lesions. More importantly, almost one third (28.2\%) of the OHPs, 'Never' informed patients that such screening assessments were taking place. No statistically significant differences were found by professional background.

After excluding those who reported 'Very rarely' conducting oral cancer screenings, for almost all OHPs (97.6\%) the examination involved a visual inspection of the patient's oral cavity and $81.1 \%$ also included an extraoral visual inspection (Table 3). Meanwhile, a screening examination that "Always" included either a visual inspection of the oropharynx or conducting neck palpation was reported at lower rates (52.3\% and 28.1, respectively).

Apart from neck palpation, which was significantly more frequently conducted among OHTs than other professional groups $(p<0.05)$, none of the other screening techniques reached statistical significance levels by OHP background. Thirty-three respondents (11.3\%), most of whom were dentists, indicated using additional diagnostic aids/tools to identify potentially malignant lesions. The most commonly utilized additional diagnostic tools included biopsies and radiographs.

When asked if respondents had ever referred a patient to an oral medicine specialist to have a lesion investigated, which subsequently was diagnosed as a malignancy, $54.2 \%$ of respondents had either never encountered such a clinical scenario, or were unsure. Of those who had referred a patient $(n=136)$, the most common intraoral site of the malignant lesion $(n=41)$ was cancer of the tongue, followed by cancer of the floor of the mouth $(n=22)$; hard palate $(n=18)$; and the lip $(n=14)$. There were also eleven cases of bone cancer. 
Table 2 Oral screening behaviors of oral health professionals in Victoria

\begin{tabular}{|c|c|c|c|c|}
\hline & $\begin{array}{l}\text { Dentists } \\
(n=242)\end{array}$ & $\begin{array}{l}\mathrm{DHs} \\
(n=45)\end{array}$ & $\begin{array}{l}\text { OHT/DTs } \\
(n=48)\end{array}$ & $\begin{array}{l}\text { Total } \\
(n=335)\end{array}$ \\
\hline \multicolumn{5}{|c|}{ How frequently do you complete a comprehensive oral cancer screening? } \\
\hline With every patient & 51.7 & 46.7 & 54.2 & 51.4 \\
\hline With $50 \%$ or more of patients & 21.9 & 22.2 & 20.8 & 21.8 \\
\hline With less than $50 \%$ of patients & 13.2 & 17.8 & 14.6 & 14.0 \\
\hline Very rarely & 13.2 & 13.3 & 10.4 & 12.8 \\
\hline \multicolumn{5}{|c|}{ How often do you discuss risk factors for oral cancer with your patients? } \\
\hline With every patient & 5.8 & 8.9 & 8.4 & 6.6 \\
\hline With $50 \%$ or more of patients & 22.7 & 40.0 & 33.3 & 26.6 \\
\hline With less than $50 \%$ of patients & 47.9 & 35.5 & 41.7 & 45.3 \\
\hline Very rarely & 23.6 & 15.6 & 16.6 & 21.5 \\
\hline \multicolumn{5}{|c|}{$\begin{array}{l}\text { What factors influence your decision to perform an oral cancer screening examination? } \\
\text { (Multiple answers) }\end{array}$} \\
\hline Patient complains of a problem & 32.2 & 31.1 & 41.4 & 33.6 \\
\hline Age of the patient & 31.4 & 25.2 & 43.8 & 32.4 \\
\hline Medical history & 26.9 & 31.1 & 43.8 & 29.9 \\
\hline Other & 16.5 & 26.6 & 25.0 & 19.1 \\
\hline
\end{tabular}

Table 3 Oral cancer screening examination behaviors of oral health professionals who performed screening more frequently than "Very rarely"

\begin{tabular}{|c|c|c|c|c|}
\hline & $\begin{array}{l}\text { Dentists } \\
(n=210)\end{array}$ & $\begin{array}{l}\mathrm{DHs} \\
(n=39)\end{array}$ & $\begin{array}{l}\text { OHT/DTs } \\
(n=43)\end{array}$ & $\begin{array}{l}\text { Total } \\
(n=292)\end{array}$ \\
\hline \multicolumn{5}{|c|}{$\begin{array}{l}\text { Screening examination involved: } \\
\text { Extra-oral visual inspection of the oral cavity }\end{array}$} \\
\hline Always & 80.4 & 82.1 & 83.7 & 81.1 \\
\hline Sometimes & 2.9 & 5.1 & 0.0 & 2.7 \\
\hline Never & 16.7 & 12.8 & 16.3 & 16.2 \\
\hline \multicolumn{5}{|c|}{ A visual inspection of the oral cavity } \\
\hline Always & 98.1 & 93.0 & 100 & 97.6 \\
\hline Sometimes & 1.4 & 7.0 & 0.0 & 2.1 \\
\hline Never & 0.5 & 0.0 & 0.0 & 0.3 \\
\hline \multicolumn{5}{|c|}{ A visual inspection of the oropharynx } \\
\hline Always & 50.5 & 64.1 & 50.0 & 52.3 \\
\hline Sometimes & 40.3 & 35.9 & 42.9 & 40.0 \\
\hline Never & 9.2 & 0.0 & 7.1 & 7.7 \\
\hline \multicolumn{5}{|l|}{ Neck palpation } \\
\hline & & & & * \\
\hline Always & 25.5 & 23.7 & 44.2 & 28.1 \\
\hline Sometimes & 56.9 & 52.6 & 32.5 & 52.6 \\
\hline Never & 17.6 & 33.7 & 21.3 & 19.3 \\
\hline
\end{tabular}

${ }^{*}$ Chi- squared test; $p$-value: 0.05
OHP respondents were asked about their clinical decision pathway once an abnormal lesion was detected and the practitioner could not reach a diagnosis. The most commonly reported courses of action included: 'Referral to an oral medicine specialist' (85.7\%); 'Follow-up at a later appointment' (53.7\%); and 'Consultation with another colleague' (45.4\%). Least mentioned courses of action included: 'Ask the patient to monitor the lesion' (38.2\%); and 'Referral to the patient's general medical practitioner' (14.3\%). Differences by professional background were statistically significant for: 'Consultation with another colleague' $(p<0.001)$, where DHs and OHT/DTs were more likely to pursue this course of action; and 'Asking the patient to monitor the lesion', whereby OHT/DTs (55.8\%) were more likely to ask the patient to monitor the lesion compared with $35.9 \%$ and $35.9 \%$ for dentists and DHs, respectively $(p<0.05)$.

Regarding knowledge of oral cancer risk factors, 99.4\% of participants identified smoking as a risk factor for oral cancer. The second most frequently identified risk factor was chewing betel nut and chewing tobacco products (98.2\%). A history of oral cancer was the third most frequently identified risk factor (97.0\%), followed by family history of oral cancer (96.1\%), and alcohol consumption (94.6\%). The oral cancer risk knowledge score ranged from 0 to 88 with a mean of 68.5 (s.d. 10.1).

When OHPs were asked how often they discussed risk factors for oral cancer with their patients, the largest group $(45.3 \%)$ indicated discussing risk factors for oral cancer with less than $50 \%$ of their patients (Table 2). Another $21.5 \%$ reported they 'Very rarely' discussed risks 
factors with their patients. Discussion of risk factors in 'More than $50 \%$ of patients' or 'With every patient' was reported by $26.6 \%$ and $6.6 \%$ of OHP respondents, respectively. No significant differences were demonstrated by professional background.

Concerning participants' level of confidence in discussing behavioral issues that may affect oral health with their patients, participants generally felt confident discussing oral hygiene practices (Mean 8.9; s.d. 1.6), diet and nutrition (Mean 8.3; s.d. 1.7), tobacco use (Mean 8.2; s.d. 1.8), and to some extent alcohol consumption (Mean 7.3; s.d. 2.2). In contrast, the mean level of confidence in discussing sexual behaviors (i.e. oral sex practices) was comparatively low at 3.6 (s.d. 1.8).

The probability of conducting an oral health screening examination ("Very rarely": 0 vs. Other categories [i.e., 'With every patient'; 'With $50 \%$ or more of patients'; and 'With less than $50 \%$ of patients']: 1) was explored utilizing LRA with age, sex, profession, rurality and number of years of work experience, risk factor knowledge index, self-assessed oral cancer knowledge, and self-confidence items as independent variables (Table 4). After controlling for the other independent variables included in the model, four variables remained statistically significant $\left[\mathrm{X}^{2}(4)=40.689 ; p<0.0001\right]$. Results indicated that dental specialists, were less likely to perform an oral cancer screening examination $(\mathrm{OR}=0.18$; $95 \% \mathrm{CI} 0.07$ to 0.52$)$. As the level of self-assessed knowledge of oral cancer increased, so too did the likelihood of performing an oral cancer screening examination (OR $=1.35$; 95\% CI 1.09 to 1.67). Additionally, when the OHP's level of confidence in discussing oral hygiene with the patient increased, the likelihood of conducting an oral cancer screening examination also increased (OR $=1.25 ; 95 \%$ CI 1.03 to 1.52$)$. On the other hand, when the 'Patient complained of a problem' the likelihood of performing an oral cancer screening examination decreased ( $\mathrm{OR}=0.21 ; 95 \% \mathrm{CI} 0.10$ to 0.44 ). The variance for oral cancer screening examination, using the full model, was $22.5 \%$ (Nagelkerke ${ }^{2} \mathrm{r}=0.225$ ).

\section{Discussion}

The present study represents an attempt to explore oral cancer-related practices and attitudes among different
OHPs working in the Australian state of Victoria. This study's results show little over 50\% of OHPs perform comprehensive oral cancer screening with all patients. Although these results are not directly comparable due to wording of questions and response categories, they would contrast with earlier studies done in Australia where OHPs routinely performed oral mucosal screening on $85-95 \%$ of their recall and new patients $[4,21]$. In the same manner, studies done elsewhere, reported that $85-89 \%$ of dentists and $66-78 \%$ of dental hygienists performed oral cancer examination for their patients, although they did not indicate frequency of performing these examinations $[29,30]$. Apart from this, the present study is largely in line with one other previous study done among DH/DT and OHTs in Australia on the importance and need for routine oral cancer screening [33].

A high proportion indicated that they did so in almost all patients older than 40 years. However, only about two-thirds of respondents routinely screened patients 20-39 years of age. The study also indicated that OHPs, who screened all patients, were less likely to always include neck palpation, and to a lesser extent, visual inspection of the oropharynx in their oral cancer screening practice. This may be due to lack of confidence in undertaking a head and neck examination as evidenced in other study done in Australia [21, 33]. These findings are cause for some concern as many oral cancers may not present with visibly detectable signs or symptoms during the pre-malignant or localized stages, which represents a clinical window in which these lesions are most amenable to treatment [29]. Such early stage lesions could otherwise be detected by a comprehensive and thorough visual examination of high risk sites $[22,42]$ and facilitating patient education about oral cancer.

Furthermore, a comprehensive oral cancer screening examination would seem essential, particularly given the growing incidence of HPV positive oropharyngeal cancers $[4,22,42,43]$. Consequently, it is important for OHPs to maintain and develop competence and confidence in neck palpation techniques and visual examination of the oropharynx as part of their oral cancer screening practices [22]. Where necessary, appropriate education programs should be developed to address the

Table 4 Regression coefficient, odds ratios and 95\% confidence interval for odds ratios for the factors associated with the probability of conducting an oral health screening examination among oral health professionals in Victoria, Australia

\begin{tabular}{llll}
\hline & $\beta$ coefficient & Odds ratio & 95\% Confidence interval \\
\hline Dental specialist & -1.70 & 0.18 & 0.07 to 0.52 \\
Self-assessed knowledge & 0.30 & 1.35 & 1.09 to 1.67 \\
Level of confidence (oral hygiene) & 0.22 & 1.25 & 1.03 to 1.52 \\
Patient complained of a problem & -1.56 & 0.21 & 0.10 to 0.44 \\
Constant & -1.04 & & \\
Nagelkerke ${ }^{2}=0.225$ & & & \\
\hline
\end{tabular}


knowledge and skill gaps in this area [4,21]. The present study showed that OHTs performed oral cancer screening somewhat more frequently than other OHP groups.

As the incidence of oral cancer continues to rise [9], the role of oral health professionals in the prevention, early identification and management of oral malignancies will become increasingly relevant to public health. In order to reduce the morbidity and mortality of oral cancer, it is necessary to implement programs aimed at prevention, early identification and diagnosis. Early identification would involve facilitating patient recognition of signs and symptoms and providing education about when to consult a health professional. Patient delays in seeking care could be reduced by self-examination for early clinical features and by population level educational interventions, particularly targeting those in the higher risk groups for oral cancer.

In recent years, awareness of cancer risk factors has increased due to wider media coverage of the issue [44]. However, studies indicate that in contrast to other types of cancers, the majority of the population are ignorant about oral cancer $[45,46]$. The public may have a tendency to interpret oral cancer symptoms and signs as relatively minor, as problems that are likely to resolve on their own. Given the lack of general public understanding of oral cancers and inability to recognise clinical symptoms and signs [22], it can be inferred that patient presentation to an OHP regarding an oral lesion may be either absent or too late, which would adversely impact on early detection and treatment outcomes. In addition to these issues, some cancers may be asymptomatic and the absence of notable symptoms may further contribute to late diagnosis.

For these reasons, opportunistic oral cancer screening examinations conducted by OHPs remain an important mode for early identification and diagnosis. Results from the multivariate analysis indicate that after controlling for other variables, dental specialists tend to perform less oral screening compared with other OHPs. It is possible that dental specialists rely on GDPs to conduct comprehensive dental examinations while they focus solely on the condition for which the patient was referred them; however, this cannot be stated categorically as the relevant information was not collected in the survey. The odds of conducting an oral screening examination also decreased when the patient complained of oral pain. It could be inferred, although once again respondents were not asked about this in the survey, that OHPs addressed the presenting complaint on these occasions (e.g. pain) and did not conduct a more comprehensive oral examination. Unsurprisingly, the data indicated that OHPs with a higher self-evaluated confidence level in oral cancer-related knowledge had greater confidence advising patients and were more likely to perform oral cancer screening examinations [21]. The ultimate goal would be to have the whole oral health team collaborate with an integrated approach to oral health care, particularly in the context of oral cancer knowledge, screening practices and patient and community education.

When considering these results, certain limitations must be acknowledged. The low response rate may have introduced non-response bias, and combined with the self-reported nature of the data means it is not possible to generalize findings to all OHPs working in Victoria, Australia. Furthermore, response rates to online surveys have been demonstrated to be less than for surveys administered using a paper-based approach $[47,48]$. However, the response rate is also related to the size of the population under study with larger populations requiring smaller response rates [48]. Thus, although the number of responses received was not as high as was hoped, the achieved overall response rate was within the expected range for online surveys among oral health professionals $(2.5 \%$ to $26 \%)[49,50]$.

Despite some minor differences, this study group is largely representative of the Victorian OHP population in terms of age, profession, gender and distribution. There was an overrepresentation of DH/DT/OHTs in the study sample, who make up $28 \%$ of total respondents but only $17 \%$ of all registered dental practitioners in Victoria [35]. When considering the socio-demographic profile, there is no available data to determine the age range of each dental practitioner group in the wider profession in the state of Victoria. However, the age profile of survey respondents (55.5\% aged 45 years or younger) is very similar that for all dental practitioners in Australia at the time of the survey ( $56.7 \%$ aged less than 45 years old) [35]. The proportion of female dentists in the study also mirrored figures reported for the state of Victoria (57\%) in 2015. Most dentists worked in urban areas (80.0\%) [51].

\section{Conclusions}

Together with risk behavior change, oral cancer screening has been shown to contribute to reductions in oral cancer mortality rates among high-risk individuals [22, 52]. It is highly probable that OHPs will see patients with oral cancer and many more with potentially malignant lesions in their professional lives $[4,21]$. Present results suggest that continued efforts to enhance the quality and consistency of oral cancer screening practices are required, which should include education and training of OHPs in up-todate and evidence-based screening methods. The recommendation to see an oral health professional at least once a year would only be beneficial if patients are routinely screening for oral cancer. The results from this study may assist in the development of oral cancer-related education and training programs for OHPs. Increased emphasis on regular training in oral cancer screening protocols and in 
patient education and counselling practices are imperative to improve oral cancer prevention and rates of early detection, to reduce the mortality-burden resulting from oral cancers.

\section{Abbreviations}

DHs: Dental Hygienists: DT: Dental Therapists; GDP: General Dental Practitioners; HNC: Head and Neck Cancer; HPV: Human Papilloma Virus; OHP: Oral Health Professional; OHTs: Oral Health Therapists; OPC: Oro-Pharyngeal Cancers

\section{Acknowledgements \\ Not applicable}

\section{Funding}

This study was partially funded by a grant received from DENTSPLY Research \& Innovation Grants.

\section{Availability of data and materials}

Ethics approvals were granted on the basis that only researchers involved in the study could access the de-identified data. Raw data have been stored securely at the Melbourne Dental School, The University of Melbourne. The minimum retention period is 5 years from publication or public release of the results. Supporting documents are available upon request to the corresponding author

\section{Authors' contributions}

RM1 contributed with the conception of the study, design, draft of the manuscript, read and approved the final manuscript. SH carried out the fieldwork, contributed with the data analysis and draft of the manuscript, and read and approved the final manuscript. RM2 contributed with the conception of the study, and the design and draft of the manuscript, and read and approved the final manuscript. DB contributed with the conception of the study, and the design and draft of the manuscript, and read and approved the final manuscript. MM1 contributed with the conception of the study, interpretation of the data and the design and draft of the manuscript. RM3 contributed with interpretation of the data and final drafting of the manuscript. BK carried out part of the fieldwork, interpretation of the data and final drafting of the manuscript. AC contributed with interpretation of the data and drafting of the manuscript. All the authors revised and approved the manuscript. MM2 contributed with the conception of the study, and the design and draft of the manuscript, and read and approved the final manuscript.

\section{Ethics approval and consent to participate}

This study was approved by The Human Research Ethics Committee of The University of Melbourne, Australia (\#1442325.1).

All participants conseted to participate in this study. It was explained to potential participants that reception of completed surveys, was assumed as consent for participation in the study.

\section{Consent for publication}

Not Applicable

\section{Competing interests}

The authors declare that they have no competing interest. RM is a Section Editor for BMC Oral Health.

\section{Publisher's Note}

Springer Nature remains neutral with regard to jurisdictional claims in published maps and institutional affiliations,

\section{Author details}

'Oral Health Cooperative Research Centre, Melbourne Dental School, University of Melbourne, Melbourne, VIC 3010, Australia. 'Department of Public Health \& Human Sciences, Preventive \& Public Health Dentistry, Fukuoka Dental College, Sawara-ku, Fukuoka, Japan. ${ }^{3}$ Melbourne Dental School, University of Melbourne, Melbourne, Australia. ${ }^{4}$ eviDent Foundation, South Yarra, Australia. ${ }^{5}$ University of Melbourne, Melbourne, Australia.
Received: 1 August 2017 Accepted: 27 November 2017

Published online: 15 December 2017

\section{References}

1. World Health Organization. Cancer Fact Sheet. http://www.who.int/ mediacentre/factsheets/fs297/en/. Accessed 24 Jun 2017.

2. Fox P, Boyce A. Cancer health inequality persists in regional and remote Australia. Med J Aus. 2014;201:445-6.

3. Ferlay J, Soerjomataram I, Dikshit R, Eser S, Mathers C, Rebelo M, Parkin DM, Forman D, Bray F. Cancer incidence and mortality worldwide: sources, methods and major patterns in GLOBOCAN 2012. Int J Cancer. 2015;136:E359-86.

4. Farah CS, Simanovic B, Dost F. Oral cancer in Australia 1982-2008: a growing need for opportunistic screening and prevention. Aust Dent J. 2014;59:349-59.

5. Australian Institute of Health and Welfare. Australian cancer incidence and mortality (ACIM) books: head and neck including lip cancer. Canberra: AlHW; 2016.

6. National Institute of Dental and Craniofacial Research. Detecting Oral Cancer: A Guide for Health Care Professionals. 2017. http://www.nidcr.nih.gov/oralhealth/ Topics/OralCancer/DetectingOralCancer.htm. Accessed 21 May 2017.

7. Young D, Xiao CC, Murphy B, Moore M, Fakhry C, Day TA. Increase in head and neck cancer in younger patients due to human papillomavirus (HPV). Oral Oncol. 2015:51:727-30.

8. Deschler DG, Richmon JD, Khariwala SS, Ferris RL, Wang MB. The "new" head and neck cancer patient-young, nonsmoker, nondrinker, and HPV positive: evaluation. Otolaryngol Head Neck Surg. 2014;151:375-80.

9. Australian Institute of Health and Welfare: Head and neck cancer statistics. https:/head-neck-cancer.canceraustralia.gov.au/statistics. Accessed 20 June 2017.

10. Warnakulasuriya S, Fennell N, Diz P, Seoane J, Rapidis A. An appraisal of oral cancer and pre-cancer screening programmes in Europe: a systematic review. J Oral Pathol Med. 2015;44:559-70.

11. Ariyawardana A, Johnson NW. Trends of lip, oral cavity and oropharyngeal cancers in Australia 1982-2008: overall good news but with rising rates in the oropharynx. BMC Cancer. 2013;13:333. doi:10.1186/1471-2407-13-333.

12. Bhatia N, Lalla Y, Vu AN, Farah CS. Advances in optical adjunctive aids for visualisation and detection of oral malignant and potentially malignant lesions. Int J Dent. 2013. doi:10.1155/2013/194029; https://www.hindawi. com/journals/ijd/2013/194029/. Accessed 21 July 2017

13. Silverman S Jr, Kerr AR, Epstein JB. Oral and pharyngeal cancer control and early detection. J Cancer Educ. 2010;25:279-81.

14. Mehrotra R, Gupta DK. Exciting new advances in oral cancer diagnosis: avenues to early detection. Head Neck Oncol. 2011;3:33.

15. Garzino-Demo P, Dell'Acqua A, Dalmasso P, Fasolis M, La Terra Maggiore GM, Ramieri G, Berrone S, Rampino M, Schena M. Clinicopathological parameters and outcome of 245 patients operated for oral squamous cell carcinoma. J Craniomaxillofac Surg. 2006;34:344-500.

16. DeSantis CE, Lin CC, Mariotto AB, Siegel RL, Stein KD, Kramer JL, Alteri R, Robbins AS, Jemal A. Cancer treatment and survivorship statistics, 2014. CA Cancer J Clin. 2014;64:252-71.

17. Onizawa K, Nishihara K, Yamagata K, Yusa H, Yanagawa T, Yoshida H. Factors associated with diagnostic delay of oral squamous cell carcinoma. Oral Oncol. 2003;39:781-8.

18. McLeod NM, Saeed NR, Ali EA. Oral cancer: delays in referral and diagnosis persist. Br Dent J. 2005;198:681-84.19.

19. Mighell AJ, Gallagher JE. Oral cancer - improving early detection and promoting prevention. Are you up to date? Br Dent J. 2012:213:297-9.

20. Grimes D, Patel J, Avery C, New NICE. Referral guidance for oral cancer: does it risk delay in diagnosis? Br J Oral Maxillofac Surg. 2016:55:404-6.

21. Allen K, Ford PJ, Farah CS. Oral mucosal screening and referral attitudes of Australian oral health therapists and dental hygienists in Queensland. Int J Dent Hyg. 2015;13:206-12.

22. Ford PJ, Farah CS. Early detection and diagnosis of oral cancer: strategies for improvement. J Cancer Policy. 2013;1:e2-7.

23. Grulich AE, Jin F, Conway EL, Stein AN, Hocking J. Cancers attributable to human papillomavirus infection. Sex Health. 2010;7:244-52.

24. Speight $\mathrm{P}$, Warnakulasuriya $\mathrm{S}$, Ogden $\mathrm{G}$. Early detection and prevention of oral cancer: a management strategy for dental practice. Br Dent J. 2010:1-37.

25. World Dental Federation. Oral cancer. https://www.fdiworlddental.org/resources/ policy-statements-and-resolutions/oral-cancer. Accessed July 262017.

26. British Dental Association. Opportunistic oral cancer screening: A management strategy for dental practice. BDA occasional paper 2000 
http://oralcancerfoundation.org/wp-content/uploads/2017/01/screening-17. pdf. Accessed July 262017.

27. Australian Dental Association. Policy Statement 6.10 - Oral Cancer Screening. 2017. https://www.ada.org.au/Dental-Professionals/Policies/Dental-Practice/610-Oral-Cancer-Screening/ADAPolicies_6-10_OralCancerScreening_V1. Accessed July 262017.

28. Gajendra S, Cruz GD, Kumar JV. Oral cancer prevention and early detection: knowledge, practices, and opinions of oral health care providers in New York state. J Cancer Educ. 2006;21:157-62.

29. LeHew CW, Epstein JB, Kaste LM, Choi YK. Assessing oral cancer early detection: clarifying dentists' practices. J Public Health Dent. 2010;70:93-100.

30. Decuseara G, MacCarthy D, Menezes G. Oral cancer: knowledge, practices and opinions of dentists in Ireland. J Ir Dent Assoc. 2011;57:209-14.

31. Vijay Kumar KV, Suresan V. Knowledge, attitude and screening practices of general dentists concerning oral cancer in Bangalore city. Indian J Cancer. 2012;49:33-8.

32. Razavi SM, Zolfaghari B, Foroohandeh M, Doost ME, Tahani B. Dentists' knowledge, attitude, and practice regarding oral cancer in Iran. J Cancer Educ. 2013;28:335-41.

33. Allen K, Farah C. Screening and referral of oral mucosal pathology: a checkup of Australian dentists. Aust Dent J. 2015;60:52-8.

34. Dost F, Do L, Farah CS. Lesion evaluation, screening and identification of oral neoplasia study: an assessment of high-risk Australian populations. Community Dent Oral Epidemiol. 2016:44:64-75. doi:10.1111/cdoe.12191. Epub 2015 Aug 11

35. Dental Board of Australia Registrant Data. 2015. http://www.dentalboard. gov.au/About-the-Board/Statistics.aspx. Accessed 14 May 2017.

36. Parashos P, Morgan MV, Messer HH. Response rate and nonresponse bias in a questionnaire survey of dentists. Community Dent Oral Epidemiol. 2005;33:9-16

37. Bogen K. The effect of questionnaire length on response rates: a review of the literature, U.S.Bureau of the Census, editor. 1996: Washington D.C. URL: https://www.census.gov/srd/papers/pdf/kb9601.pdf. Accessed 03 Mar 2017.

38. Edwards P, Roberts I, Clarke M, DiGuiseppi C, Pratap S, Wentz R, Kwan I. Increasing response rates to postal questionnaires: systematic review. $\mathrm{Br}$ Med J (Clin Res Ed). 2002;324:1183.

39. Scott A, Jeon SH, Joyce CM, Humphreys JS, Kalb G, Witt J, Leahy AA. Randomised trial and economic evaluation of the effect of response mode on response rate, response bias, and item non-response in a survey of doctors. BMC Med Res Methodol. 2011;11:12.

40. Cohen J. Statistical power analysis for the behavioral sciences. Hillside, NJ: Lawrence Erlbaum Assoc. Inc; 1988.

41. Australia Post. Local delivery service. URL: https:/auspost.com.au/content/dam/ auspost_corp/media/documents/post-guides/post-charges-guidems 11 . pdf?fm=search-organic. Accessed 12 Dec 2017.

42. Mashberg A, Samit A. Early diagnosis of asymptomatic oral and oropharyngeal squamous cancers. CA Cancer I Clin. 1995;45:328-51.

43. Gillison ML, D'Souza G, Westra W, Sugar E, Xiao W, Begum S, Viscidi R. Distinct risk factor profiles for human papillomavirus type 16-positive and human papillomavirus type 16-negative head and neck cancers. J Natl Cancer Inst. 2008;100:407-20.

44. Eliott J, Forster AJ, McDonough J, Bowd K, Crabb S. An examination of Australian newspaper coverage of the link between alcohol and cancer 2005 to 2013. BMC Public Health. 2017;18:47.

45. Mariño R, Minichiello V, Wright C, Schofield M. Oral health beliefs and practices among Greek and Italian older Australians: a focus group approach. Australas J Ageing. 2002;21:193-8.

46. Williams M, Bethea J. Patient awareness of oral cancer health advice in a dental access centre: a mixed methods study. Br Dent J. 2011;210:E9-E9.

47. Nulty D. The adequacy of response rates to online and paper surveys: what can be done? Assess Eval High Educ. 2008;33:301-14.

48. Funkhouser E, Fellows JL, Gordan W, Rindal DB, Foy PJ, Gilbert GH. Supplementing online surveys with a mailed option to reduce bias and improve response rate: the National Dental Practice-Based Research Network. J Public Health Dent. 2014;74:276-82.

49. Goodchild JH, Donaldson M. The use of sedation in the dental outpatient setting: a web-based survey of dentists. Dent Implantol Updat. 2011;22:73-800.

50. Henry RK, Molnar A, Henry JCA. Survey of US dental practices' use of social media. J Contemp Dent Pract. 2012;13:137-41.

51. Australian Institute of Health and Welfare. Dental workforce 2011. Canberra: Australian Government: Australian Institute of health and Welfare. p. 2013.

52. Sankaranarayanan R, Ramadas K, Thara S, Muwonge R, Thomas G, Anju G, Mathew B. Long term effect of visual screening on oral cancer incidence and mortality in a randomized trial in Kerala, India. Oral Oncol. 2013;49:314-21.

\section{Submit your next manuscript to BioMed Central and we will help you at every step:}

- We accept pre-submission inquiries

- Our selector tool helps you to find the most relevant journal

- We provide round the clock customer support

- Convenient online submission

- Thorough peer review

- Inclusion in PubMed and all major indexing services

- Maximum visibility for your research

Submit your manuscript at www.biomedcentral.com/submit 